\title{
Natural widths of hypersatellite K-X-ray lines and lifetimes of double K-hole states in mid-Z atoms
}

\author{
J. Rzadkiewicz ${ }^{\text {a,* }}$, D. Chmielewska ${ }^{\text {a }}$, Z. Sujkowski $^{\text {a }}$, M. Berset ${ }^{\text {b }}$, \\ J.-Cl. Dousse b, Y.-P. Maillard b, O. Mauron b, P.-A. Raboud b, M. Polasik ${ }^{\text {c }}$, \\ K. Słabkowska ${ }^{\mathrm{c}}$, J. Hoszowska ${ }^{\mathrm{d}}$, M. Pajek ${ }^{\mathrm{e}}$ \\ a Andrzej Soltan Institute for Nuclear Studies, 05-400 Swierk, Poland \\ ${ }^{\mathrm{b}}$ Physics Department, University of Fribourg, CH-1700 Fribourg, Switzerland \\ c Faculty of Chemistry, Nicholas Copernicus University, 87-100 Toruń, Poland \\ ${ }^{\mathrm{d}}$ European Synchrotron Radiation Facility ESRF, F-38043 Grenoble, France \\ e Institute of Physics, Akademia Świętokrzyska, 25-406 Kielce, Poland
}

Available online 24 May 2005

\begin{abstract}
K-X-ray spectra of $\mathrm{Zr}, \mathrm{Nb}$, Mo and Pd targets bombarded with $250 \mathrm{MeV}$ carbon and $360 \mathrm{MeV}$ oxygen ions are studied with high resolution diffraction spectrometry. Relative yields and natural widths of the $\mathrm{K}^{\mathrm{h}} \alpha_{1,2}$ hypersatellite lines are determined and compared with those of the diagram lines. The widths of $K^{\mathrm{h}} \alpha_{1,2}$ lines are compared with the new phenomenological formula which takes into account the retardation of the $\mathrm{K}^{\mathrm{h}} \alpha_{1}$ transition in the LS coupling scheme. The data significantly exceed the expected values. Deduced lifetimes of the double K-hole states are shorter than expected.
\end{abstract}

PACS: $32.30 . \mathrm{Rj} ; 32.70 .-\mathrm{n} ; 32.70 . \mathrm{Jz} ; 34.50 . \mathrm{Fa}$

Keywords: K-X-ray hypersatellites; Line shapes and linewidths; Relative intensities; MCDF calculations

\section{Introduction}

K-X-ray hypersatellite spectra originate from transitions where the initial state has two vacancies in the K-shell. They were discovered in the begin-

\footnotetext{
* Corresponding author. Fax: +4822 7793481.

E-mail address: jacek@iriss.ipj.gov.pl (J. Rzadkiewicz).
}

ning of the seventies [1]. Ever since, they remain in the focus of very special interest to both basic [2] and applied atomic physics [3]. Firstly, the hypersatellite K-X-ray spectra are particularly suitable to study the Breit interaction, one of the most elusive and least studied of all atomic interactions [4]. Furthermore, the strengths of the $\mathrm{K}^{\mathrm{h}} \alpha_{1}$ and $\mathrm{K}^{\mathrm{h}} \boldsymbol{\alpha}_{2}$ hypersatellite transitions are determined by the 
coupling scheme dominating in the atom (see Fig. 1). The de-excitation of the $\mathrm{K}^{-2}$ hole state via the spin flip $\mathrm{K}^{\mathrm{h}} \alpha_{1}$ transition is forbidden in the pure LS coupling scheme $\left({ }^{1} \mathrm{~S}_{0} \rightarrow{ }^{3} \mathrm{P}_{1}\right)$ and fully allowed in the $j j$ coupling. As a result, the intensity ratio $R=I\left(\mathrm{~K}^{\mathrm{h}} \alpha_{1}\right) / I\left(\mathrm{~K}^{\mathrm{h}} \alpha_{2}\right)$ differs considerably from that of the corresponding diagram lines which is almost constant and equals two. This ratio is thus a sensitive quantitative measure of the coupling which changes with the atomic number from the LS coupling limit at low $\mathrm{Z}$ to the $j j$ coupling limit at high $Z$. Another coupling-dependent quantity is the natural width of the hypersatellite line, which gives direct information on the lifetimes of the so-called hollow atoms, which are characterized by the shortest lifetimes of any bound atomic states [5]. Hollow atoms became a focus of interest lately as model systems for atoms far from stability, useful for studies of ultrafast dynamics in chemistry, biology, and materials science [6]. They have also been proposed as a way of achieving the population inversion and the lasing for hard X-ray lasers [3].

The present paper focuses on the natural widths of the K-X-ray hypersatellite transitions of mid-Z atoms induced in collisions with fast carbon and oxygen ions. The measured widths are compared

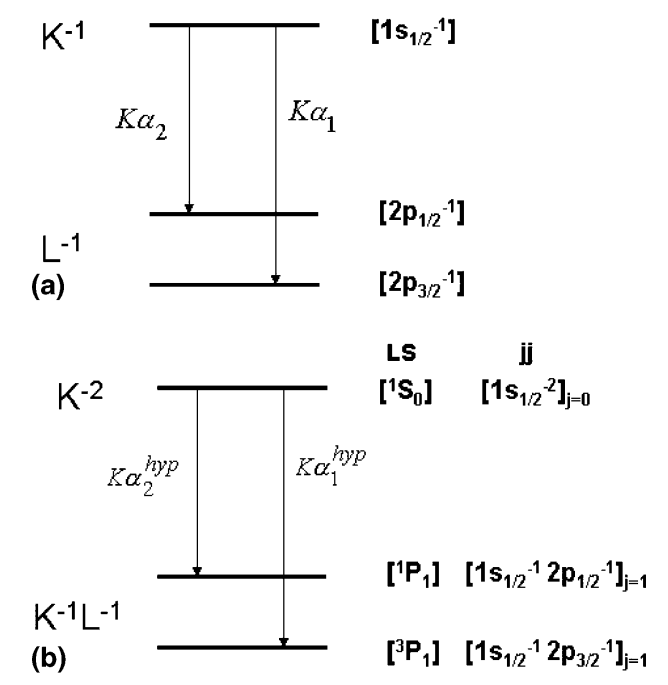

Fig. 1. Energy diagram showing the decay of (a) singly and (b) double $\mathrm{K}$-shell ionized states via $\mathrm{K} \alpha_{1,2}$ diagram and $\mathrm{K}^{\mathrm{h}} \alpha_{1,2}$ hypersatellite X-ray transitions, respectively. with the theoretical predictions based on the MCDF calculations and phenomenological formulas.

\section{Experiment and data analysis}

The experiments were performed at the variable energy cyclotron of the Paul Scherrer Institute (PSI), in Villigen, Switzerland. The highly charged ${ }^{12} \mathrm{C}^{5+}$ and ${ }^{16} \mathrm{O}^{7+}$ ions delivered by a $10 \mathrm{GHz} \mathrm{CA}$ PRICE ECR source were accelerated to the energy of $250 \mathrm{MeV}$ and $360 \mathrm{MeV}$, respectively. Beam currents of 200-750 nA were used, depending on the investigated elements.

The high-resolution measurements of the $\mathrm{K} \alpha$ spectra were performed with an on-line transmission-type curved crystal spectrometer [7]. A $0.15 \mathrm{~mm}$-wide rectangular slit, located on the Rowland circle, $2 \mathrm{~cm}$ in front of the target, served as the effective source of radiation. In this modified DuMond geometry the line shapes of the observed transitions are almost insensitive to the beam-induced thermal deformations of the target. A schematic drawing of the experimental set-up is given in Fig. 2.

The targets consisted of $4 \times 40 \mathrm{~mm}^{2}$ self-supporting metallic foils with thicknesses of $31.7 \mathrm{mg}$ / $\mathrm{cm}^{2}$ for $\mathrm{Zr}, 9.1 \mathrm{mg} / \mathrm{cm}^{2}$ for $\mathrm{Nb}, 26.4 \mathrm{mg} / \mathrm{cm}^{2}$ for Mo and $25.9 \mathrm{mg} / \mathrm{cm}^{2}$ for Pd. They were mounted on a special aluminium holder and tilted to $15^{\circ}$ with respect to the target-crystal direction. This angle was chosen as a compromise between the self-absorption of the X-rays in the target and the target area seen by the crystal through the slit, which had an opposite dependence on the tilt angle.

A $1 \mathrm{~mm}$ thick $\mathrm{SiO}_{2}(110)$ crystal $(2 d=$ $2.456671 \AA$ ) was used for the diffraction of the X-rays. The crystal plate was bent cylindrically to a radius of $313 \mathrm{~cm}$ and the effective reflecting area was about $5 \times 5 \mathrm{~cm}^{2}$. The Bragg angles were measured by means of an optical laser interferometer with a precision of about $0.01 \mathrm{arcsec}$. The diffracted X-rays were detected with a 3 in. diameter $\times 1 \mathrm{~mm}$-thickness $\mathrm{NaI}$ scintillator surrounded by an anti-Compton ring. Good events were selected by setting energy-windows in the 


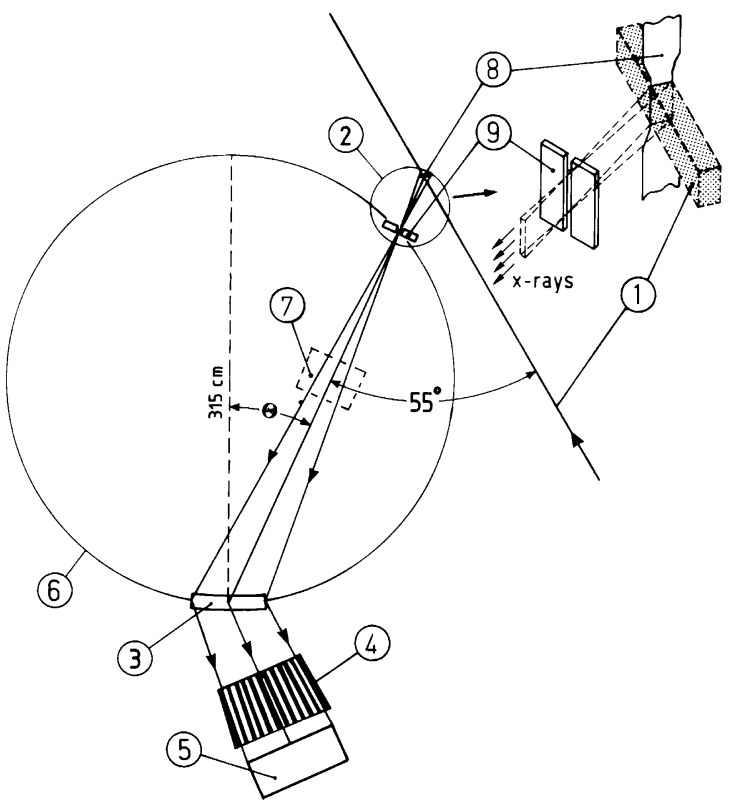

Fig. 2. Schematic diagram of the DuMond slit geometry used in the present experiment (not to scale): (1) beam, (2) target chamber, (3) crystal, (4) collimator, (5) detector, (6) focal circle, (7) monitor detector, (8) target and (9) tantalum slit. $\Theta$ represents the Bragg angle.

ADC spectra. The energy windows were adjusted automatically by the acquisition program as a function of the Bragg angles. The heavy-ion beam intensity was monitored by means of a $13 \mathrm{~mm}^{2} \mathrm{Si}$ PIN photodiode viewing the target through the same slit as the crystal of the spectrometer. The data were collected for each Bragg angle until a preset number of $\mathrm{K} \alpha$-X-rays was obtained in the monitor detector so that each point of the highresolution spectra corresponded to the same number of collisions.

The energy calibration of the spectra was based on the photoinduced $\mathrm{K} \alpha_{1}$ diagram lines measured for each target on both sides of reflection. The photoionization was produced by irradiating the targets with the bremsstrahlung from a $\mathrm{Au}$ anode Coolidge X-ray tube equipped with a $1 \mathrm{~mm}$ thick $\mathrm{Be}$ window and operated typically at $60 \mathrm{kV} \times 50 \mathrm{~mA}$.

All spectra were collected in first order on the right side of reflection by step scanning over the given angular region. In order to survey the stability of the experimental set-up, the spectra were measured in several successive scans. For illustration, the measured $\mathrm{K} \alpha-\mathrm{X}$-ray spectrum of molybdenum induced by $20 \mathrm{MeV} / \mathrm{amu}$ carbon ions is shown in Fig. 3.

The profile of an X-ray line resolved with a crystal spectrometer can generally be represented by the convolution of a Lorentzian and a Gaussian, which results in the so-called Voigt profile. The Lorentzian represents the natural line shape of the X-ray transition, while the Gaussian profile accounts for the instrumental response of the spectrometer and the additional broadening due to the multiple ionization in the outer shells $[8,9]$. The observed spectral lines were analysed by means of a least-squares-fitting program employing Voigt functions. The instrumental response could be extracted from the measurements of the $\mathrm{K} \alpha_{1}$ diagram lines in the photoinduced X-ray spectra. The determination of the additional broadening due to the multiple ionization was a little more complicated. Assuming that the degree of multiple ionization is the same for diagram and hypersatellite lines, the broadening could be obtained by comparison of the widths of the $\mathrm{K} \alpha_{1}$ diagram lines in the photoinduced and heavy-ions induced X-ray spectra. The fixed Gaussian widths employed in

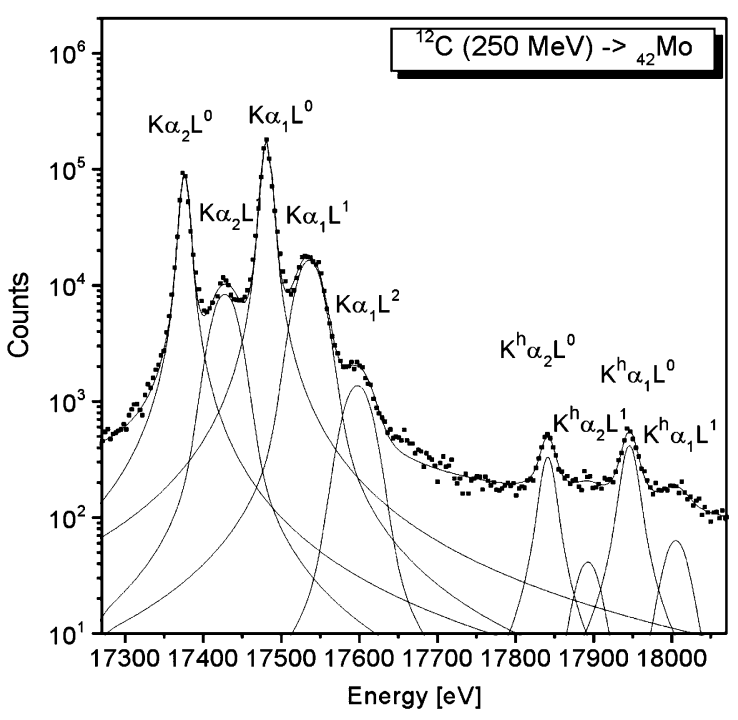

Fig. 3. Measured $\mathrm{K} \alpha$-X-ray spectrum of molybdenum induced by $20 \mathrm{MeV} / \mathrm{amu}$ carbon ions. $\mathrm{L}^{n}$ indicates the transition with $n$ spectator vacancies in the L-shell. 
the fits of the hypersatellites correspond thus to the instrumental response corrected for the unresolved satellites contaminations (see Section 3). The centroid energies, intensities and Lorentzian widths of the hypersatellite lines were let free in the fitting procedure. The measured hypersatellite yields were corrected for the X-ray absorption in the target.

\section{Effect of $L$ - and M-shell ionization on the K-X-ray spectra}

The $\mathrm{K} \alpha \mathrm{L}^{1,2}$ satellites of the diagram $\mathrm{K} \alpha \mathrm{L}^{0}$ lines as well as $\mathrm{K}^{\mathrm{h}} \alpha \mathrm{L}^{1}$ satellites of the hypersatellite $\mathrm{K}^{\mathrm{h}} \alpha \mathrm{L}^{0}$ lines are well resolved in the spectrum (Fig. 3). The $\mathrm{K}^{\mathrm{h}} \alpha \mathrm{L}^{0}$ lines correspond to the deexcitation of initial states with two K-shell holes and a closed L-shell (i.e. He-like hole states [10]). The M-shell satellites due to the additional M-shell ionization cannot be resolved from the $\mathrm{K} \alpha \mathrm{L}^{0}$ nor the $\mathrm{K}^{\mathrm{h}} \alpha \mathrm{L}^{0}$ lines because the energy shifts are smaller than the natural widths. No statement about the M-shell closure can be therefore made.

The M-shell ionization produces a broadening and an energy shift of the X-ray lines. These effects increase with the square of the atomic number of the projectile. They are at maximum when the projectile velocity matches that of the M-shell electrons of the target atom. The average numbers of the M-shell holes at the moment of the emission of the diagram and hypersatellite X-ray lines were assumed to be approximately the same. Actually, MCDF calculations show that the average energy shifts of diagram and hypersatellite lines due to one additional $\mathrm{M}$-shell hole are almost the same (maximum difference is $\sim 0.12 \mathrm{eV}$ ) [10], whereas two additional $\mathrm{M}$-shell holes cause different average shifts of the $\mathrm{K} \alpha$ and $\mathrm{K}^{\mathrm{h}} \alpha$ lines (maximum difference is $\sim 1.3 \mathrm{eV}$ ). Therefore, if the M-shell ionization probability is low, one can find a correction for the broadening of the X-ray lines by the determination of the Gaussian widths (corresponding to the instrumental response and broadening due to the multiple M-shell ionization) of the diagram $\mathrm{K} \alpha_{1}$ lines in the heavy-ions induced $\mathrm{X}$-ray spectra. Only such corrected experimental values can be compared with the theoretical results obtained for transitions in which the M-shell
Table 1

Gaussian widths $[\mathrm{eV}]$ of the heavy-ion-induced $\mathrm{K} \alpha_{1}$ lines

\begin{tabular}{lll}
\hline Element & ${ }^{12} \mathrm{C}(250 \mathrm{MeV})$ & ${ }^{16} \mathrm{O}(360 \mathrm{MeV})$ \\
\hline${ }_{40} \mathrm{Zr}$ & $7.3(0.4)$ & $11.6(0.4)$ \\
${ }_{4} \mathrm{Nb}$ & $8.8(0.3)$ & $13.7(0.3)$ \\
${ }_{42} \mathrm{Mo}$ & $8.9(0.2)$ & $12.6(0.3)$ \\
${ }_{46} \mathrm{Pd}$ & $10.8(0.4)$ & $16.8(0.4)$ \\
\hline
\end{tabular}

ionization is not considered in the initial and final vacancy configuration states. For this purpose, independent studies of the M-shell ionization probabilities for the investigated collisions with carbon ions have been performed [11]. In these studies, the following average numbers of spectator M-shell holes at the moment of the K-X-ray emission were obtained: 1.08(20), 1.04(26), $0.98(17), 0.86(11)$ for $\mathrm{Zr}, \mathrm{Nb}$, Mo and Pd, respectively. The low M-shell ionization probabilities allowed us thus to determine the broadening corrections of the hypersatellite lines from the Gaussian widths obtained from the fits of the diagram lines. The Gaussian widths of the $\mathrm{K} \alpha_{1}$ diagram lines obtained in the X-ray spectra induced by impact with $250 \mathrm{MeV}$ carbon and $360 \mathrm{MeV}$ oxygen ions are presented in Table 1.

\section{Results and discussion}

The lifetime $\tau$ of a single hole in an atomic level is related to the natural width $\Gamma$ of that level by the uncertainty principle:

$\Gamma=\hbar / \tau$,

where $\hbar$ is the reduced Planck constant. Assuming that the de-excitation processes are independent, the total level width (natural width) is given by

$\Gamma=\Gamma_{\mathrm{r}}+\Gamma_{\mathrm{n}}$,

where $\Gamma_{\mathrm{r}}$ and $\Gamma_{\mathrm{n}}$ are radiative and non-radiative widths, respectively. Knowing the natural widths of the initial $\left(\Gamma_{\mathrm{i}}\right)$ and final $\left(\Gamma_{\mathrm{f}}\right)$ atomic levels, one can obtain the natural width of the corresponding $\mathrm{X}$-ray transition:

$\Gamma(\mathrm{i} \rightarrow \mathrm{f})=\Gamma_{\mathrm{i}}+\Gamma_{\mathrm{f}}$.

The natural widths of the $\mathrm{K}_{1,2}\left(\mathrm{~K}^{-1} \rightarrow L_{\mathrm{II}, \mathrm{III}}^{-1}\right)$ diagram lines (see Fig. 1) can be written as 
$\Gamma\left(K \alpha_{1,2}\right)=\Gamma_{\mathrm{K}}+\Gamma_{\mathrm{L}_{\mathrm{IIIIII}}}$,

where $\Gamma_{\mathrm{K}}$ and $\Gamma_{\mathrm{L}_{\mathrm{IIIII}}}$ are the natural widths of the $1 \mathrm{~s}^{-1}$ and $2 \mathrm{p}^{-1}$ atomic states. The natural widths of the $\mathrm{K}^{-1}$ atomic states increases from $0.03 \mathrm{eV}$ $\left(\tau_{\mathrm{K}}=2.2 \times 10^{-14} \mathrm{~s}\right)$ for ${ }_{3} \mathrm{Li}$ [12] to $95.7 \mathrm{eV}\left(\tau_{\mathrm{K}}=\right.$ $\left.6.9 \times 10^{-18} \mathrm{~s}\right)$ for ${ }_{92} \mathrm{U}$ [13]. The experimental natural widths of $K \alpha_{1,2}$ lines are known with high accuracy up to high $Z$ atoms (with errors of few eV) [13]. On the contrary, data for hypersatellite widths are rather scarce and not consistent. Due to the double vacancy state in the K-shell, the natural linewidths of hypersatellites are expected to be bigger than the ones of the corresponding diagram lines. Assuming that all decay channels are allowed (jj coupling), the width of the initial state $\mathrm{K}^{-2}$ can be estimated by

$\Gamma_{\mathrm{i}}=\Gamma_{\mathrm{KK}} \approx 2 \Gamma_{\mathrm{K}}$

and the width of the final state $\mathrm{K}^{-1} \mathrm{~L}^{-1}$ by

$\Gamma_{\mathrm{f}}=\Gamma_{\mathrm{KL}} \approx \Gamma_{\mathrm{K}}+\Gamma_{\mathrm{L}}$.

The natural width of a hypersatellite line can thus be written as [14]:

$\Gamma\left(\mathrm{K}^{\mathrm{h}} \alpha_{1,2}\right) \approx 3 \Gamma_{\mathrm{K}}+\Gamma_{\mathrm{L}_{\mathrm{III}, \mathrm{II}}}$.

The $j j$ coupling assumed above dominates only for high- $Z$ atoms. In the case of mid- $Z$ atoms, theoretical calculations of linewidths have to take into account the intermediate coupling, in which the $\mathrm{K}^{\mathrm{h}} \alpha_{1}$ is only partly allowed. This effect should enlarge the lifetime of the $\mathrm{K}^{-2}$ hole state and correspondingly decrease the natural width of the hypersatellite line. Taking into account the intermediate coupling and assuming that the fluorescence yields $\omega_{\mathrm{KK}}$ are the same in the LS and $j j$ coupling schemes, the width of the $\mathrm{K}^{-2}$ hole state can be rewritten as

$\Gamma_{\mathrm{KK}}=\left(2-\frac{4}{3} \kappa_{\mathrm{LS}}\right) \Gamma_{\mathrm{K}}$,

where $\kappa_{\mathrm{LS}}$ is a parameter varying from 0 to 1 , corresponding to the "strength" of the LS coupling with respect to the $j j$ coupling. The parameter $\kappa_{\mathrm{LS}}$ can be determined from the measured intensity ratio $R=\mathrm{K}^{\mathrm{h}} \alpha_{1} / \mathrm{K}^{\mathrm{h}} \alpha_{2}$, using the following relation:

$\kappa_{\mathrm{LS}}=1-\frac{R}{2}$.
For the intermediate coupling, the natural widths of the hypersatellites can thus be written as

$\Gamma\left(\mathrm{K}^{\mathrm{h}} \alpha_{1,2}\right)=\left(3-\frac{4}{3} \kappa_{\mathrm{LS}}\right) \Gamma_{\mathrm{K}}+\Gamma_{\mathrm{L}_{\mathrm{IIIII}}}$.

In Fig. 4 the experimental natural widths $\Gamma_{1}$ and $\Gamma_{2}$ of the $\mathrm{K}^{\mathrm{h}} \alpha_{1}$ and $\mathrm{K}^{\mathrm{h}} \alpha_{2}$ lines are compared with values calculated with the phenomenological laws corresponding to the $j j$ coupling (Eq. (7)) (solid line) and intermediate coupling (Eq. (10)) (dashed line), respectively. The single vacancy level widths were taken from [15]. Results of MCDF calculations concerning ${ }_{30} \mathrm{Zn}$ and ${ }_{36} \mathrm{Kr}$ [16] are also presented in Fig. 4. As shown, the experimental data significantly exceed the phenomenological values for intermediate coupling. The lifetimes of the double K-hole states $\tau_{\mathrm{KK}}$ shown in Fig. 5 were deduced from the natural widths $\Gamma_{\mathrm{KK}}$. The latter were obtained directly from the averaged differences between the fitted Lorentzian widths of the $\mathrm{K} \alpha_{1,2}$ hypersatellite and diagram lines measured in the heavy-ions induced X-ray spectra. The experimental lifetimes are close to those predicted by the phenomenological law for $j j$ coupling (solid
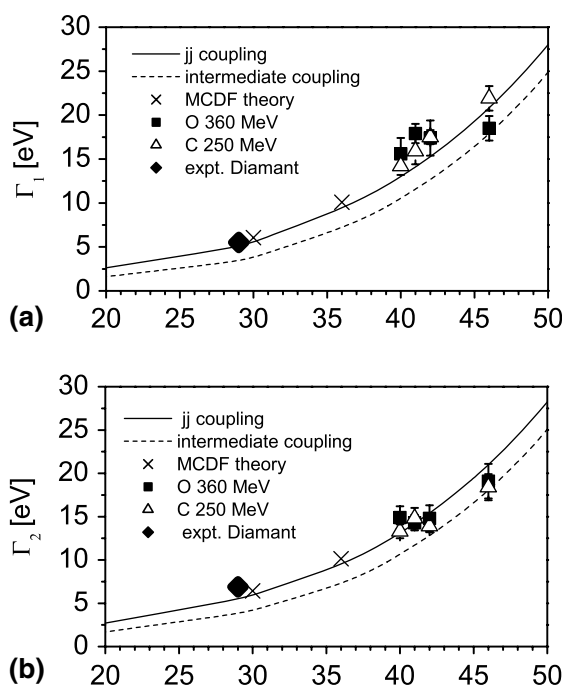

Fig. 4. $\Gamma_{1}$ (a) and $\Gamma_{2}$ (b) natural widths of the $\mathrm{K}^{\mathrm{h}} \alpha_{1}$ and $\mathrm{K}^{\mathrm{h}} \alpha_{2}$ lines compared with the results given by the phenomenological formulas based on the $j j$ coupling (solid line) and intermediate coupling (dashed line), respectively. $\mathrm{X}$ represents results of MCDF calculations performed for $\mathrm{Zn}$ and $\mathrm{Kr}$ [16], experimental data taken from [5]. 


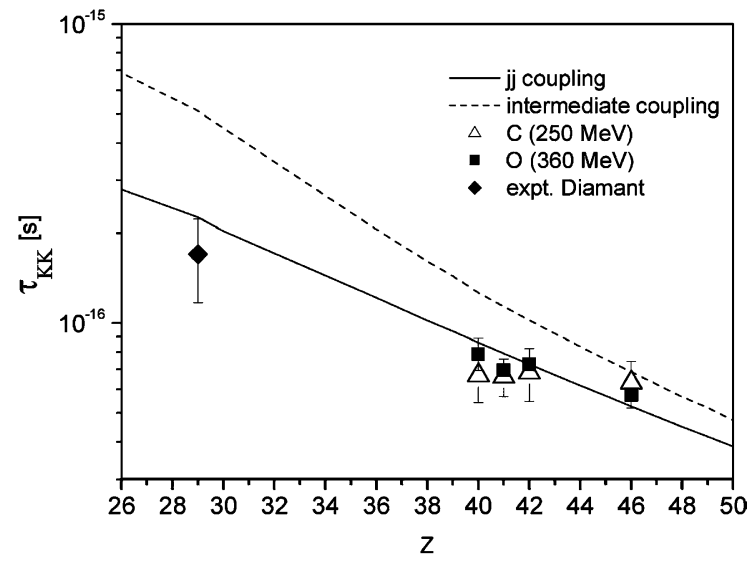

Fig. 5. Experimental double $\mathrm{K}$ shell vacancy lifetimes compared with the values obtained from the phenomenological formulas based on the $j j$ coupling (solid line) and the intermediate coupling (dashed line). ( $\diamond$ represents experimental data taken from [5].

line in Fig. 5) but significantly shorter than the values determined by means of the phenomenological law based on the intermediate coupling (dashed line), although the latter should be the most appropriate coupling scheme for the investigated mid-Z atoms.

There is no obvious mechanism which could be responsible for this apparent "speeding up" of the hypersatellite transitions. The possible changes in the fluorescence yields have been considered by Chen [16] and found to be relatively small. Any major change in the coupling scheme due to the additional M-shell hole also seems unlikely. Precise experimental data as well as detailed theoreti- cal calculations are thus clearly needed, particularly for light elements, i.e. for those elements which are governed by the LS coupling.

\section{References}

[1] J.P. Briand, P. Chevallier, M. Tavernier, J.P. Rozet, Phys. Rev. Lett. 27 (1971) 777.

[2] E.P. Kanter, R.W. Dunford, B. Krässig, S.H. Southworth, Phys. Rev. Lett. 83 (1999) 508.

[3] K. Moribayashi, A. Sasaki, T. Tajima, Phys. Rev. A 59 (1999) 2732

[4] M.H. Chen, B. Crasemann, H. Mark, Phys. Rev. A 25 (1982) 391.

[5] R. Diamant, S. Huotari, K. Hämäläinen, R. Sharon, C.C. Kao, M. Deutsch, Phys. Rev. Lett. 91 (2003) 193001;

R. Diamant, S. Huotari, K. Hämäläinen, R. Sharon, C.C. Kao, M. Deutsch, Phys. Rev. A 62 (2000) 052519-1.

[6] K. Moribayashi, A. Sasaki, T. Tajima, Phys. Rev. A 58 (1998) 2007.

[7] B. Perny, J.-Cl. Dousse, M. Gasser, J. Kern, R. Lanners, Ch. Rhême, W. Schwitz, Nucl. Instr. and Meth. A 267 (1988) 120.

[8] K.W. Hill, B.L. Doyle, S.M. Shafroth, D.H. Madison, R.D. Deslattes, Phys. Rev. A 13 (1976) 1334.

[9] B. Boschung et al., Phys. Rev. A 51 (1995) 3650.

[10] J. Rzadkiewicz et al., Phys. Lett. A 264 (1999) 186.

[11] J. Rzadkiewicz et al., Phys. Rev. A 68 (2003) 032713.

[12] P.H. Citrin, G.K. Wertheim, Phys. Rev. B 16 (1977) 4256.

[13] E.G. Kessler Jr., R.D. Deslattes, D. Girard, W. Schwitz, L. Jacobs, O. Renner, Phys. Rev. A 26 (1982) 2696.

[14] J.P. Mosse, P. Chevallier, J.P. Briand, Z. Phys. A 322 (1985) 207.

[15] J.L. Campbell, T. Papp, At. Data Nucl. Data Tables 77 (2001) 1 .

[16] M.H. Chen, Phys. Rev. A 44 (1991) 052519-1. 